\title{
Dual Stimuli-Responsive Peptide-Based Palladium Nano-lychee Spheres for Synergistic Anti-tumor Therapy
}

Xuwu Zhang ${ }^{1,2 \#, ~ T i a n ~ Y i n ~}{ }^{1,3 \#, ~ S h u a i ~ W a n g}{ }^{1}$, Zining Hao ${ }^{1}$, Yaqian He ${ }^{l}$, Chunhui Li $^{1}$, Qianqian Zhao ${ }^{1}$, Hongyu He $e^{1}$, Dawei Gao ${ }^{1,2 *}$

${ }^{1}$ Applying Chemistry Key Lab of Hebei Province, Department of Bioengineer, Yanshan University, No.438 Hebei Street, Qinhuangdao, 066004, P. R. China.

${ }^{2}$ State Key Laboratory of Metastable Materials Science and Technology, Yanshan University, Qinhuangdao 066004, P. R. China.

${ }^{3}$ Hebei Province Asparagus Industry Technology Research Institute, Qinhuangdao, P. R. China.

*Corresponding author: Prof. Dawei Gao, Tel: (+86)13930338376. E-mail: dwgao@ysu.edu.cn

${ }^{\#}$ Xuwu Zhang and Tian Yin have the same contribution for the paper. 


\section{Calculation of photothermal conversion efficiency}

The photothermal conversion efficiency $(\eta)$ of Lan-PdNPs can be calculated as follows $\eta=\frac{h \mathrm{~A} \Delta T_{\max }-\mathrm{Q}_{\mathrm{S}}}{I\left(1-10^{-A_{\lambda}}\right)}$

Where $\eta$ is the conversion efficiency from the 808-nm laser energy to thermal energy, $h$ is the heat transfer coefficient of Lan-PdNPs, A is the area cross section of irradiation, $\Delta T_{\max }$ is the steady-state maximum temperature change, $\mathrm{Q}_{\mathrm{S}}$ is the heat associated with the light absorbance of the solvent, which was measured using solvent without NPs, $I$ is the incident laser power, $A_{\lambda}$ is the absorbance of Lan-PdNPs at 808 nm.

In this equation, the value of $h \mathrm{~A}$ is unknown for calculation. In order to get $h \mathrm{~A}$, the variable $\theta$ was introduced

$\theta=\frac{T-T_{a m b}}{\Delta T_{\max }}$

Where $T$ is the solution temperature, $T_{a m b}$ is the ambient room temperature. The value of $h \mathrm{~A}$ can be derived from the following

$h A=\frac{m C_{p}}{\tau}$

Where $m$ and $C_{p}$ is the mass and heat capacity of water, respectively. During the cooling process, the thermal time constant $\tau$ can be calculated as follows

$t=-\tau \ln (\theta)$

It is obvious that the photothermal conversion efficiency can be easily calculated when the thermal time constant $\tau$ are obtained. 
Figure S1

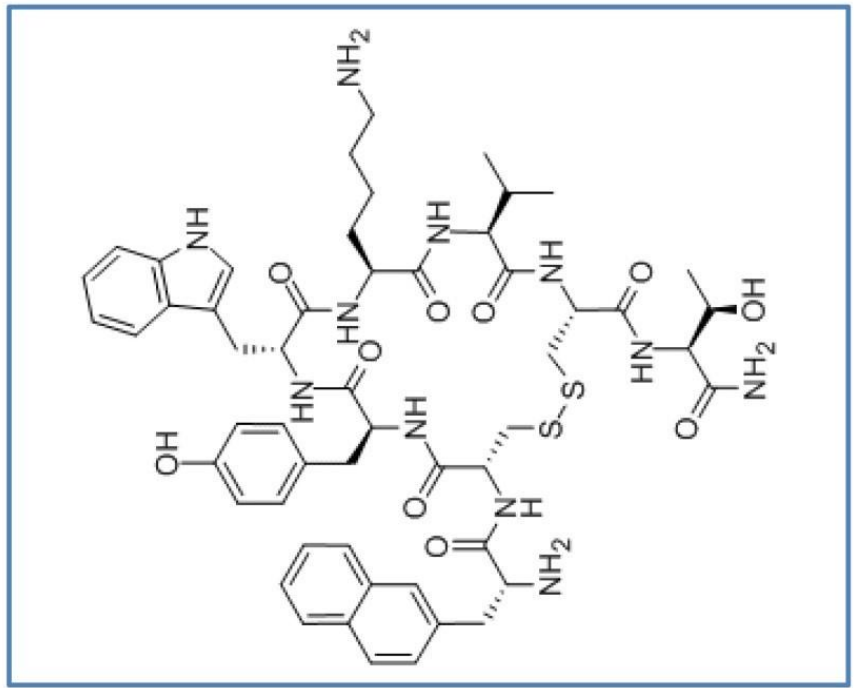

Figure S1. Structure diagram of lanreotide. 


\section{Figure S2}
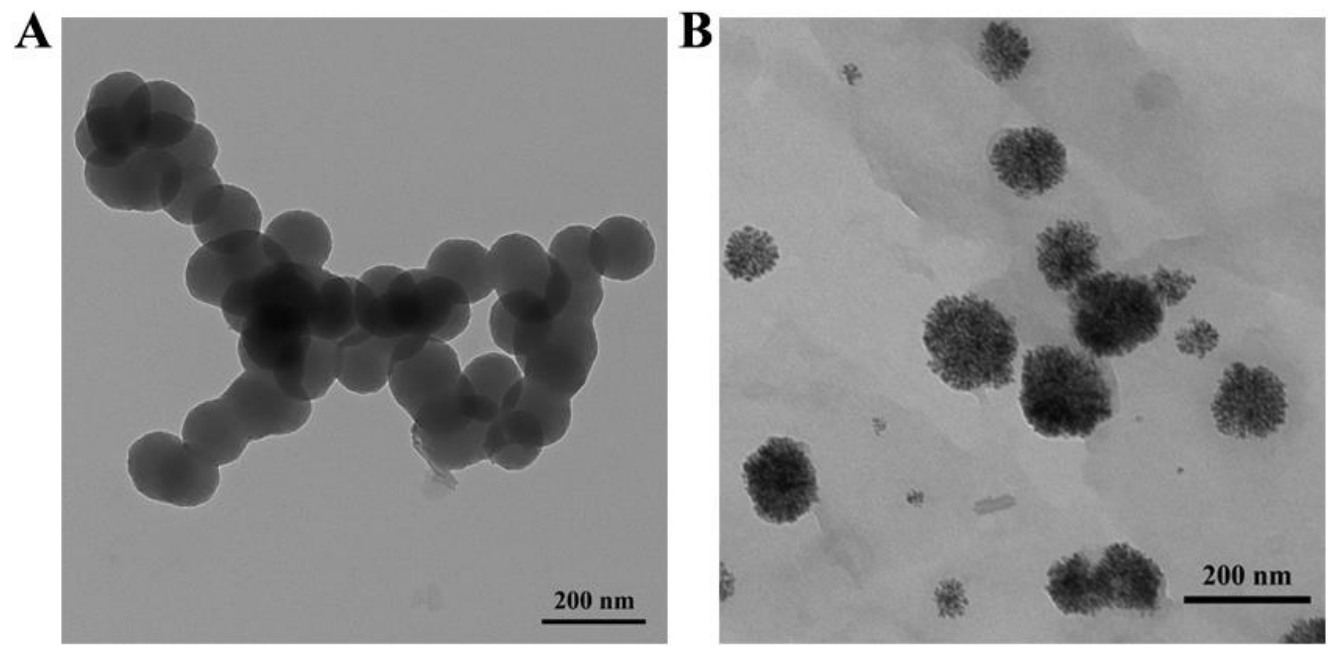

Figure S2. TEM image of (A) Lan and (B) Lan- $\left[\mathrm{PdCl}_{4}\right]^{2-}$. 
Figure S3

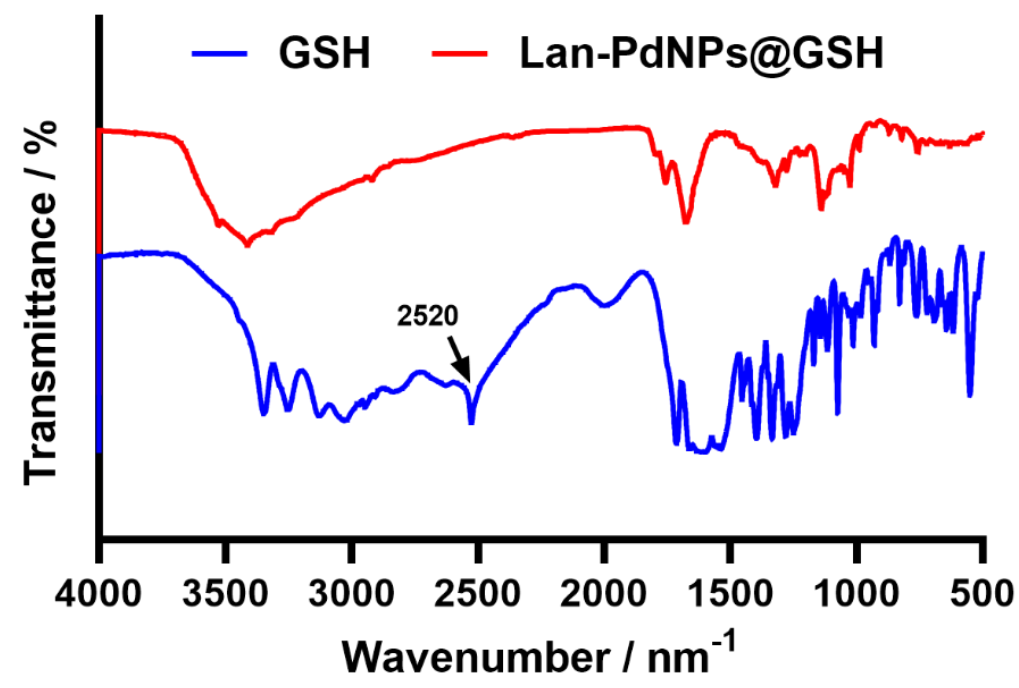

Figure S3. FTIR of GSH, and Lan-PdNPs@GSH. 
Figure S4

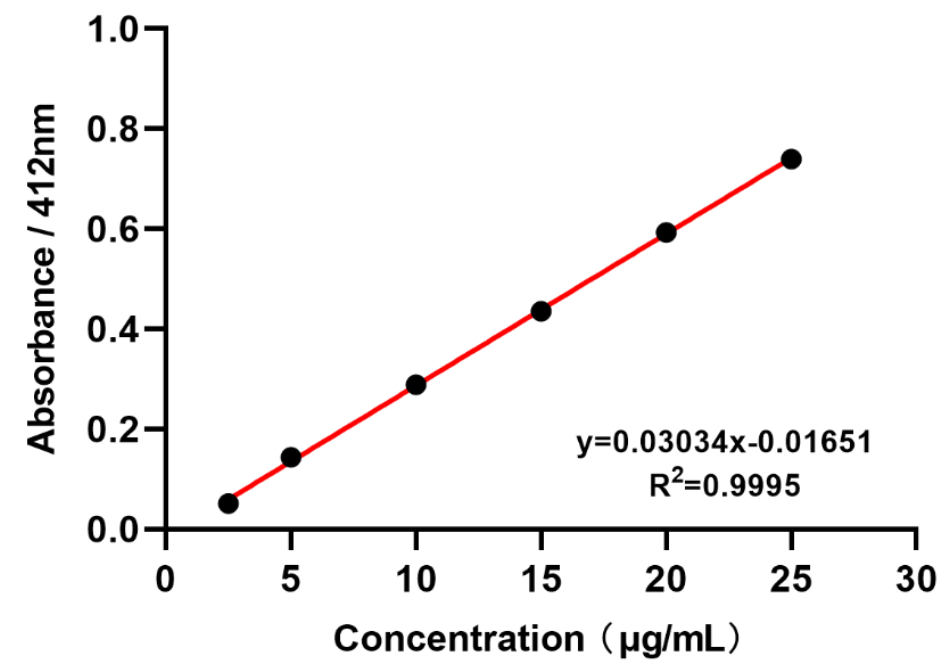

Figure S4. The standard curve of GSH with different concentrations. 


\section{Figure S5}

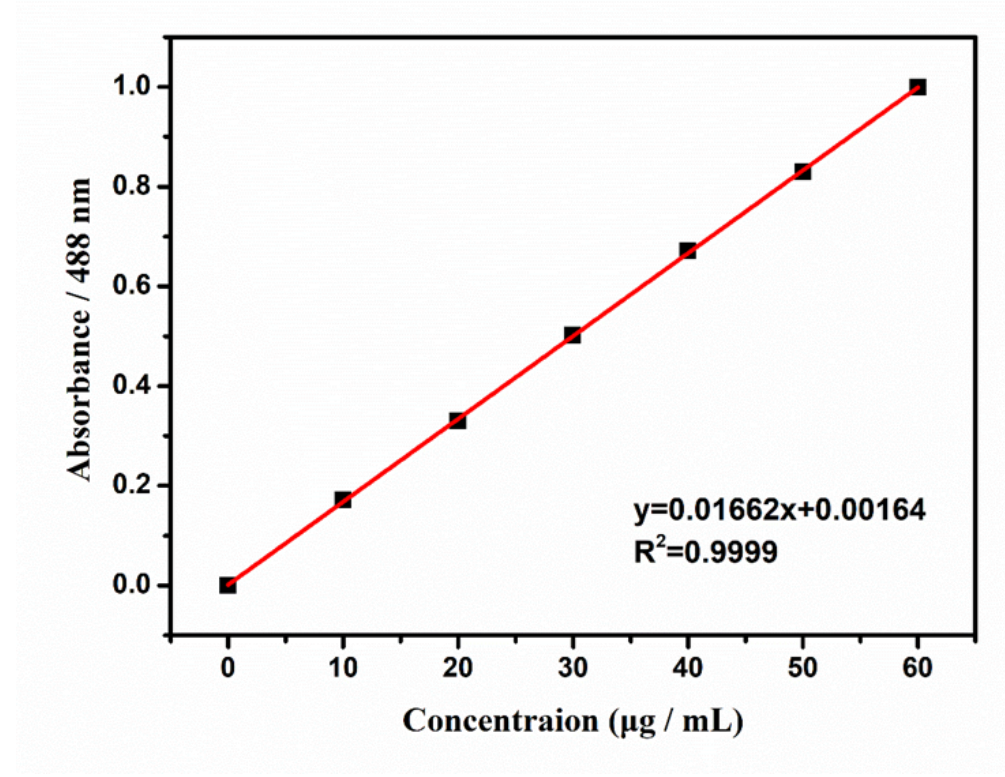

Figure S5. The standard curve of DOX with different concentrations. 


\section{Figure S6}
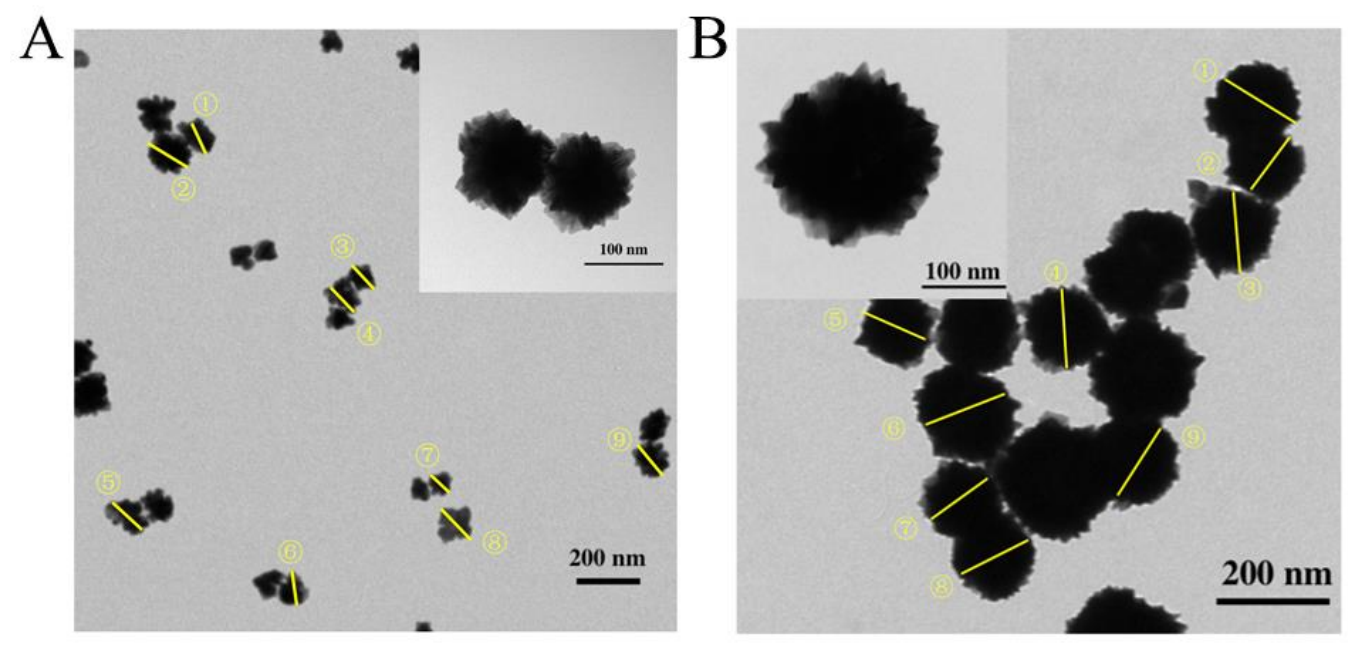

Figure S6. TEM image of samples, (A) Lan-PdNPs, (B) Lan-PdNPs@GSH/DOX.

As shown in Figure S5, we chose nine nanoparticles randomly and measured the size by ImageJ. The sizes of Lan-PdNPs were $112.2 \mathrm{~nm}, 151.0 \mathrm{~nm}, 102.0 \mathrm{~nm}, 116.8$ $\mathrm{nm}, 145.6 \mathrm{~nm}, 109.9 \mathrm{~nm}, 83.6 \mathrm{~nm}, 144.4 \mathrm{~nm}$ and $120.7 \mathrm{~nm}$. The sizes of LanPdNPs@GSH/DOX were 144.0 nm, 119.7 nm, 143.1 nm, 147.4 nm, 116.6 nm, 156.8 $\mathrm{nm}, 125.5 \mathrm{~nm}, 135.6 \mathrm{~nm}$ and $156.8 \mathrm{~nm}$. By computation, the average sizes of LanPdNPs and Lan-PdNPs@GSH/DOX were 120.7 $\pm 7.5 \mathrm{~nm} \mathrm{~nm} \mathrm{and} 138.4 \pm 5.0 \mathrm{~nm}$, respectively. 


\section{Figure S7}

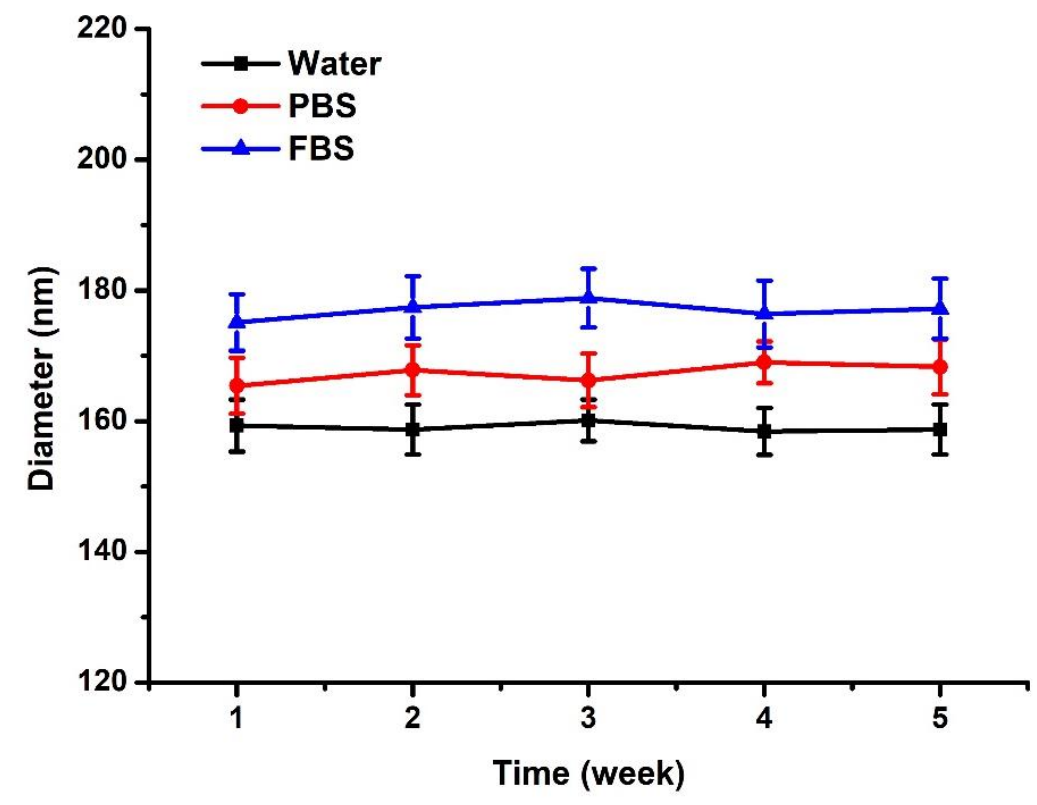

Figure S7. Stability of the Lan-PdNPs@GSH/DOX in different mediums. 


\section{Figure S8}
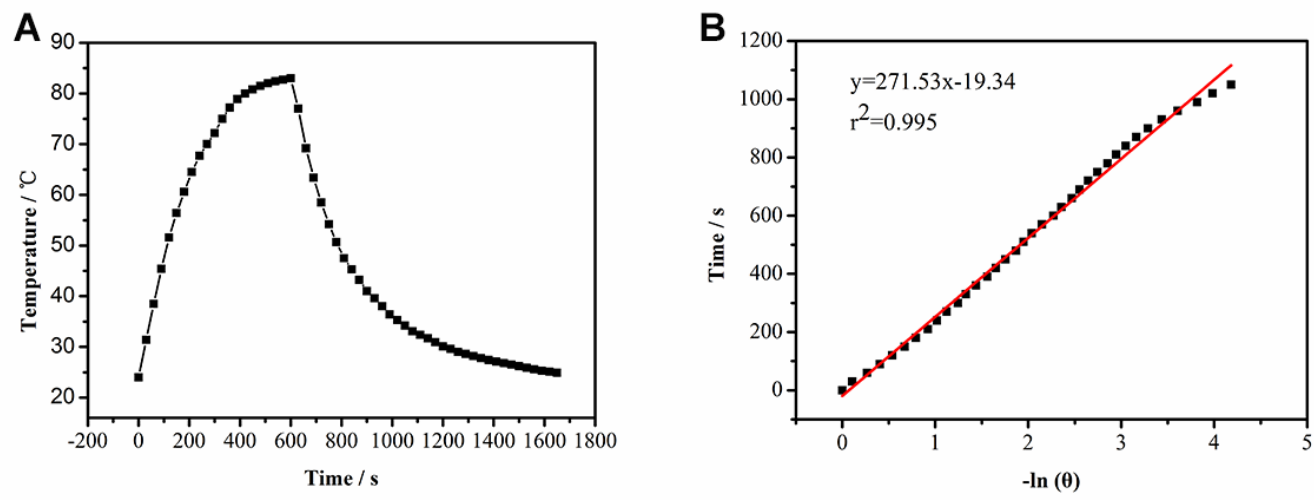

Figure S8. (A) Temporal temperature variation of the aqueous solution of LanPdNPs@GSH/DOX. The solution is irradiated by a $2 \mathrm{~W} / \mathrm{cm}^{2} 808 \mathrm{~nm}$ laser until reaching the steady temperature, and cooled to room temperature under ambient environment. (B) A linear relationship between cooling time and temperature based on the cooling curve in (A). 
Table S1 IC50 values of different formulations

\begin{tabular}{cc}
\hline Formulation & IC50 $(\mu \mathrm{g} / \mathrm{mL})$ \\
\hline Lan-PdNPs & 108.0 \\
Lan-PdNPs@GSH & 330.8 \\
Lan-PdNPs@GSH+Laser & 12.66 \\
Lan-PdNPs@GSH/DOX & 91.55 \\
Lan-PdNPs@GSH/DOX+Laser & 4.18 \\
Free DOX & 30.88 \\
\hline
\end{tabular}




\section{Figure S9}

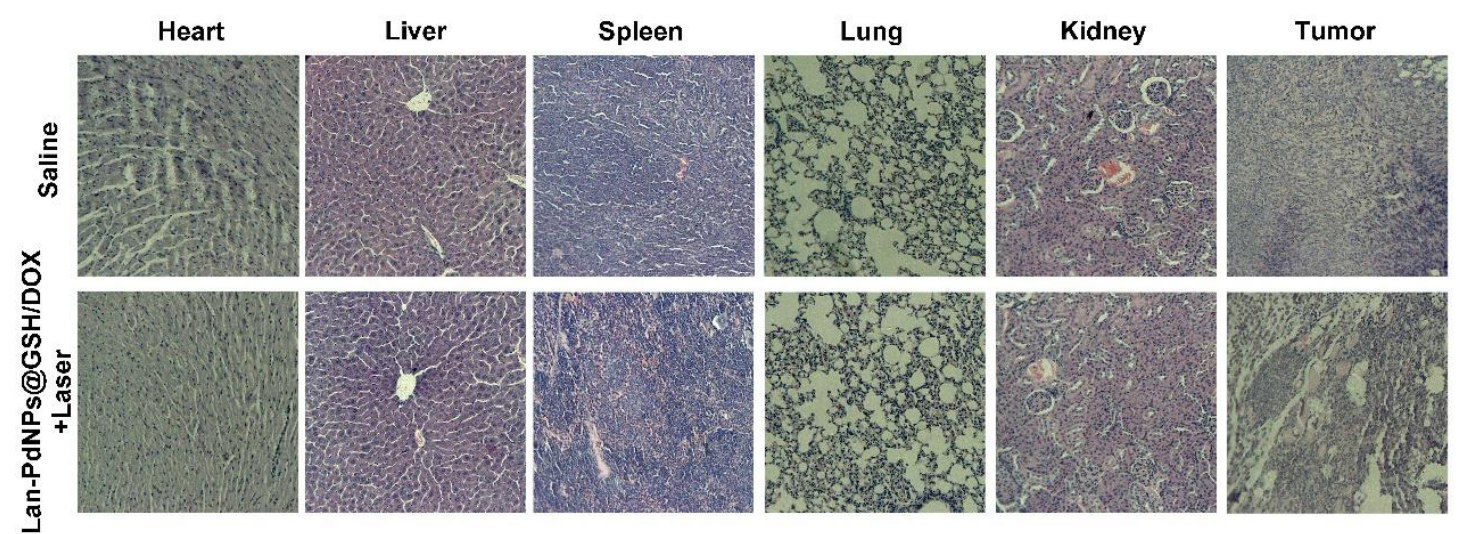

Figure S9. H\&E-stained images of the heart, liver, spleen, lung, kidney, and tumor in mice after treated by Lan-PdNPs@GSH/DOX with laser irradiation for 13 days. 


\section{Figure S10}

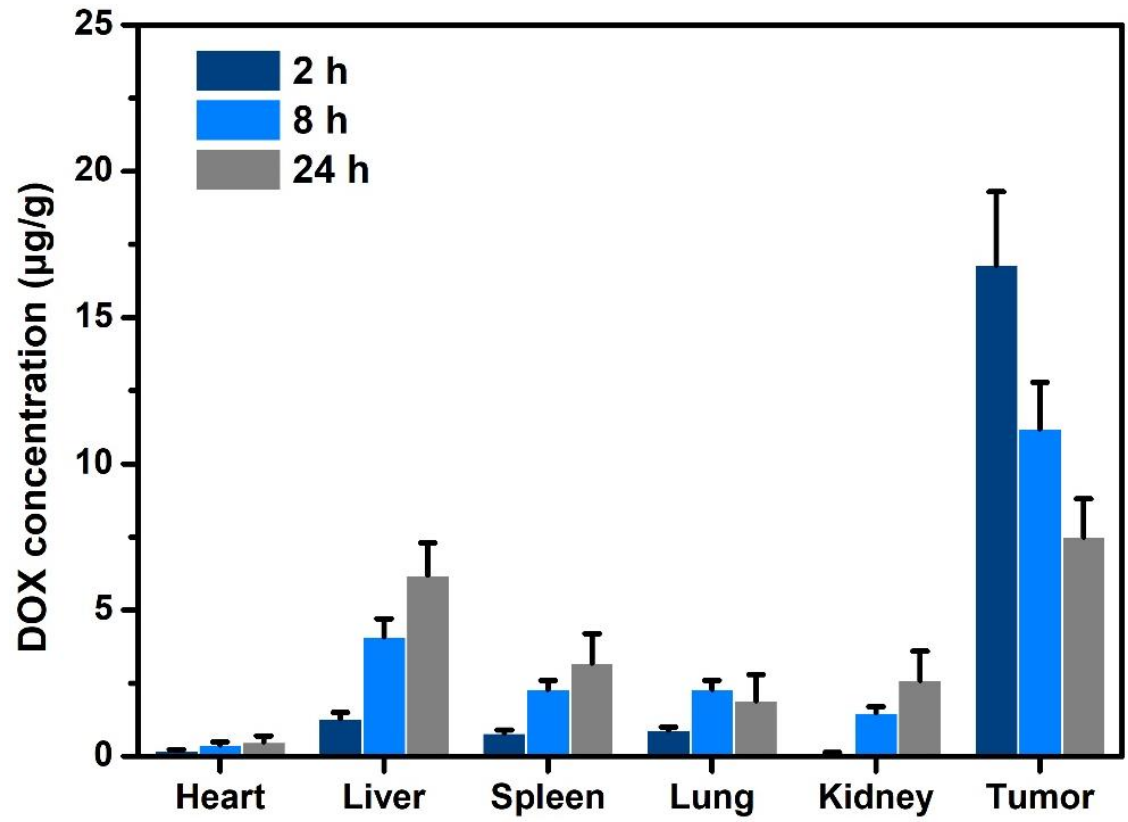

Figure S10. DOX concentrations in different tissues at $2 \mathrm{~h}, 8 \mathrm{~h}$ and $24 \mathrm{~h}$ after intratumoral injection of Lan-PdNPs@GSH/DOX.

Tumor-bearing mice were randomly assigned to three groups $(n=3)$, and injected with $200 \mu \mathrm{L}$ Lan-PdNPs@GSH/DOX intratumorally. At 2 h, 8 h and 24 h after injection, mice were sacrificed, and primary organs including heart, liver, spleen, lung, kidney and tumor were excised. Tissue specimens were washed with saline and weighed. The DOX contents were determined by the UV analysis. Briefly, tissue samples (200 mg) were first suspended in $1 \mathrm{~mL}$ of saline and homogenized with tissue homogenizer. Then, transfer $0.5 \mathrm{~mL}$ homogenate into $1.5 \mathrm{~mL}$ microtube, and the same volume of acetonitrile was added and mixed, the resulting sample was centrifuged for $10 \mathrm{~min}$ at $10000 \mathrm{rpm}$ and the supernatant was detected via UV-Vis absorption spectrophotometer. 